\title{
1 Absence of heterosis in hybrid crested newts
}

2

3 Running title - Absence of heterosis in hybrid newts

4

5 Authors and addresses

6 Jan W. Arntzen ${ }^{1}$, Nazan Üzüm ${ }^{2}$, Maja D. Ajduković ${ }^{3}$, Ana Ivanović ${ }^{1,4}$, Ben Wielstra ${ }^{1,5,6}$

$7 \quad 1$ - Naturalis Biodiversity Center, P.O. Box 9517, 2300 RA Leiden, The Netherlands

82 - Department of Biology, Faculty of Arts and Sciences, Adnan Menderes University, 09010 Aydın, Turkey

93 -Institute for Biological Research "Siniša Stanković", University of Belgrade, 11000 Belgrade, Serbia

104 -Institute of Zoology, Faculty of Biology, University of Belgrade, 11000 Belgrade, Serbia

115 - Department of Animal and Plant Sciences, University of Sheffield, S10 2TN Sheffield, United Kingdom

126 - Department of Ecology and Evolutionary Biology, University of California, Los Angeles, CA 90095, US A

13

14 Key words - age distribution, contact zone, hybrid zone, Serbia, skeletochronology, SNP markers

15

16 Corresponding author - J. W. Arntzen, Naturalis Biodiversity Center, P.O. Box 9517, 2300 RA Leiden,

17 The Netherlands. Telephone 00-31-71-5271921, E-mail pim.arntzen@naturalis.nl 


\section{Abstract}

20 We investigated relationshipsRelationships between phylogenetic relatedness, hybrid zone spatial

21 structure, the amount of interspecific gene flow and population demography were investigated, with the

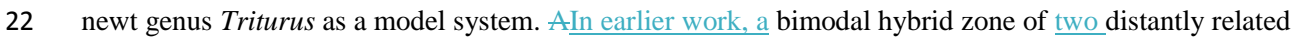

23 species in France combined low interspecific gene flow with hybrid sterility and heterosis 2 whereas a suite

24 of unimodal hybrid zones in closely related Triturus showed more or less extensive introgressive

25 hybridization with no evidence for heterosis. We here report on population demography and interspecific gene flow in two Triturus species (T. macedonicus and T. ivanbureschi in Serbia) that are moderately related, engage in a heterogeneous uni-/bimodal hybrid zone and hence represent an intermediate situation. The This study withused 13 diagnostic nuclear genetic markers $\ominus$ fin a population at the species contact zone. This showed that all individuals were hybrids, with no parentals detected. Age, size and

30 longevity and the estimated growth curves are as in purenot exceeding that of the parental species

31 pepulations, so that we conclude to the absence of heterosis in T. macedonicus - T. ivanbureschi.

32 Observations across the genus support the hypothesis that fertile hybrids allocate resources to reproduction and infertile hybrids allocate resources to growth. Several Triturus species hybrid zones not 


\section{Introduction}

47 Hybrid zones are regions where genetically distinct populations meet and hybridize (Barton and Hewitt, 1985; Harrison, 1993). They provide natural settings for the study of speciation. In particular they allow research on the consequences of new, previously untested genetic combinations of differentiated genomes in nature. Hybrid zones may take several forms, from long and narrow zones to large areas of overlap and mosaics (Arnold, 1992). The general positive relationship between the degree to which hybridization proceeds and the phylogenetic relatedness of the species involved is well established (Jiggins and Mallet, 2000). In spite of some variation in the strength of reproductive isolation for individuals from spatially isolated populations, or among different species pairs from the same evolutionary lineages (Seehausen et al., 2014; Kearns et al., 2018) accumulated evidence accumulatessuggests that, in diploid animals, reproductive isolation increases and introgression between lineages decreases with divergence (Singhal and Moritz, 2013; Arntzen et al., 2014; Beysard and Heckel, 2014; Dufresnes et al., 2014; Montanari et al., 2014; Taylor et al. 2014). Unfortunately, much less is known about how species' ecological preferences - and with that species distributions and local spatial configurations - may affect hybridization

60 and vice versa, and how phylogenetic relatedness affects the interaction with ecology. Yet, such

61 knowledge is relevant for the understanding of hybrid zones and the evolutionary inferences we draw 
from them. As Barton and Hewitt (1985) proposed ' $\ldots$ as soon as the loss of fitness through hybridization inside the zone becomes small enough ... the zone will collapse into broad sympatry', se. Accordingly, the more closely related hybridizing species are, the more hybridization will take place, over yet smaller areas. A negative relationship between the leveldegree of hybridization and the amount range overlap was indeed noted by Zuiderwijk (1980) in a variety of European amphibian species pairs but geographical variation and ecological differences remain to be studied.

We propose that a good group to investigate if reproductive isolation accumulates gradually would fulfill the following requirements. It would i) be monophyletic and ii) show more or less contiguous species ranges where iii) closely as well as distantly related species engage in hybrid zones and iv) ecological profiles of the species are different. Finally, v) to reduce the impact of spatial scale a low dispersal capability would be an asset. One group that qualifies is the European newt genus Triturus.

The genus is diverse and has a pan-European distribution, with outer ranges up to the Atlantic and the Mediterranean and reaching into Scandinavia, Russia, the Caucasus and Iran. The species group inner ranges form a patchwork and all nine species are, in one place or the other, involved in intra-generic hybridization (Arntzen et al., 2014; Wielstra et al., 2014b). The genus Triturus is composed of three clades, namely the T. marmoratus group (or marbled newts) with two species (clade A), the T. cristatus group with four species (clade B) and the T. karelinii group with three species (clade C). Clades A and B engage in western Europe and clades B and C meet up in southeastern Europe (Arntzen, 2003; Wielstra et al., 2014b). See Figure 1 for species distributions and phylogenetic relationships.

The A and B clades are distantly related with an estimated 27.6 Ma period of lineage independence (Wielstra and Arntzen, 2011). At their hybrid zone in France, T. marmoratus (clade A) and T. cristatus (clade B) interspecies $\mathrm{F}_{1}$ hybrids are infrequent (ca. $4 \%$ of the total population) and introgression is rare $(<0.1 \%$, Arntzen et al., 2009). The Heterosis is the increased vigour in a cross $\underline{\text { between two genetically differentiated lines as compared with either of the parental lines. The T. cristatus }}$ - T. marmoratus $\mathrm{F}_{1}$ hybrids show hybrid vigour in body size and longevity, that is, they get older and larger and-older than their parents (Francillon-Vieillot et al., 1990; Figure $22 \mathrm{~A}$ ). The $\underline{\text { T. cristatus }-T \text {. }}$
Commented [MJD<1]: Add page number after year Hewitt (1985: 663) 
marmoratus hybrid zone is bimodal, i.e. with predominantly the parental species and few hybrids, with a well-understood ecological differentiation. Forested and hilly areas are mostly occupied by $T$. marmoratus and open and flat areas have T. cristatus (Schoorl and Zuiderwijk, 1981; Visser et al., 2017). Representatives of clades B and C - together known as crested newts - show an intermediate level of phylogenetic relatedness with 10.4 Ma of lineage independence. TheEuropean species involved in the contact are T. cristatus, T. dobrogicus and T. macedonicus in clade B and T. ivanbureschi in clade C. Among these four, T. dobrogicus stands out as a lowland species (Vörös et al., 2016). The species meet up in the Balkan peninsula along the lower Danube and in a wide zone running from Belgrade (Serbia) to Thessaloniki (Greece) (Figure 1). Species within the B and C clades have 8.8 - 5.3 Ma of lineage independence and engage in a variety of more or less unimodal hybrid zones-across Europe, i.e. with a majority of hybrids and Asiafew or no parentals (Arntzen et al., 2014 and Wielstra et al., 2017ab), with no evidence for heterosis (hybrid vigor forso far. For data reviews in Triturus see Arntzen $-(2000 ;)$ and Lukanov and Tzankov-_(2016).

\section{Materials and methods}

Post-metamorphic crested newts were eellectedcaught with funnel traps from March to July 2013 in a pond near the village Vlasi in the southeast of Serbia (43.00 N, $22.64 \mathrm{E}$, altitude $468 \mathrm{~m}$ a.s.1.). The focal newt population we henceforth refer to as 'Vlasi'. A morphological classification on the basis of $\underline{\underline{I n} \text { total, }}$ 336 individuals were measured and marked and a small part of the newt's tail at its tip was taken for genetic analyses (Arntzen et al., 1999). The population sample was classified in five groups determined from external morphology and colouration characteristics (Arntzen and with documented phenotypes as a starting point (Wallis, 1999)wasinfive groups as and Amtzen, 1989; Amtzen \& Wallis, 1999; Amtzen, 2003; Wielstraetal., 2013b). Classes were as follows: group 1 - T. macedonicus-like (group 1,N=26), group 2-leaning towards T. macedonicus (group 2,N=48), angroup 3 - intermediate phenotype (group 3, $\mathrm{N}=146$ ), group 4 -a phenotype leaning towards T. ivanbureschi (group

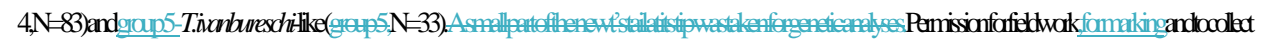


tissue samples was obtained from the Ministry of Energy, Development and Environmental Protection of the Republic of Serbia (permit no. 353-01- 35/2013-08).

Molecular data were gathered for a panel of nuclear encoded SNP markers (see below). We included reference samples with $\mathrm{N}=3$ for seven populations of $T$. ivanbureschi and seven populations of T. macedonicus available from Wielstra et al. (20132013a) (Table 1). Reference populations were located to the south of the Vlasi population, to stay awaydistant from other, more northerly distributed Triturus species. Wielstra et al. (2014a) produced sequence data for 52 short (ca. 140 bp) nuclear markers positioned in 3'UTR regions of protein-coding genes for three individuals from four populations positioned throughout the ranges of both T. ivanbureschi and T. macedonicus. We focussed on the subset of 24 nuclear markers with species diagnostic allele variants for T. ivanbureschi and T. macedonicus. Additionally an mtDNA SNP (nd4) was designed from Sanger sequence data taken from Wielstra et al. (20132013a). We determined diagnostic SNPs by checking the sequence alignments by eye in MacClade 4.08 (Maddison and Maddison, 2005).

Genotyping was conducted commercially at the SNP genotyping facility of the Institute of Biology, Leiden University, using the Kompetitive Allele-Specific PCR (KASP) genotyping system (LGC KBioscience, UK). This involves fluorescence-based genotyping using SNP-specific primers. We used the program Kraken to design two forward or reverse primers that are specific for (i.e. with a final base complementary to) one of the two potential SNP variants, in addition to a general reverse or forward primer (Semagn et al., 2014). We genotyped 378 crested newts in total: 21 individuals of both parental species (the eight populations on which SNP identification was based plus an additional six populations) and 336 individuals from Vlasi. Sequence alignments and Kraken input for all markers are available in Suppl. Inf. 1. Raw output of the KASP genotyping protocol is in Suppl. Inf. 2. The Ion Torrent nextgeneration sequence data of Wielstra et al. (2014a) used for SNP discovery are available from Dryad Digital Repository entry http://dx.doi.org/10.5061/dryad.36775. The genetic and morphological data used in the analysis are presented in Suppl. Inf. 3 and 4. 
Arntzen 7

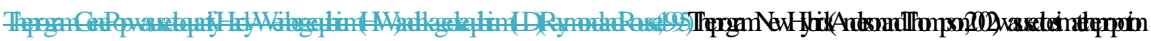

of the Vlasi population consisting of recently formed hybrids. Reference populations were invoked with the 'z-option'-, otherwise settings were default. The program HIest was used to estimate heterozygosity $\underline{(\mathrm{H})}$ and ancestry (S) (Fitzpatrick, 2012). To evaluate the consistency and independence of the genetic data and to assess hybrid zone structure Hardy-Weinberg equilibrium (HW) and linkage disequilibrium (LD) were quantified with GenePop (Raymond and Rousset, 1995).

All animals encountered were marked by clipping the middle toe on the right foot for the purpose of a population size estimate by the capture-recapture method, with results not here reported. In a subsample of individuals ( $\mathrm{N}=170,77$ males, 85 females plus eightand eight small individuals with secondary sexual characters not expressed, i.e. juveniles) that represented the five morphological groups, a phalanx of the third toe was taken for age determination by skeletochronology. Lines of arrested growhgrowth (LAGs) were counted as in Francillon-Viellot et al. (1990) and weEndosteal resorption was estimated by comparing the diameters of eroded marrow cavities of adults with the diameters of noneroded marrow cavities of juveniles and was observed in 75 males (97.4\%), 72 females (91.7\%) and two juveniles. Individual ages were estimated taking endosteal resorption into account. We also determined age at maturity from 'rapprochement'. This is the tightening of LAGs that is associated with the shift of resources from growth to reproduction. Rapprochement was not In some cases we observed a line formed at metamorphosis. Some examples are in 13 individuals $(8.0 \%)$. Figure 3.

Body size was measured in $\mathrm{mm}$ from the tip of the snout up to the posterior end of the cloaca (snout vent length, SVl). Sexual dimorphism was estimated with the Lovich and Gibbons (1992) sexual $\underline{\text { dimorphism index SDi }=\text { (mean length of the larger sex / mean length of the smaller sex) minus unity, and }}$ arbitrarily defined as positive when females are larger than males and negative in the reverse case. Von

Bertalanffy growth curves were determined with the function 'growthmodels' in the R package FSA (FSA, 2017).R Core Team, 2013; FSA, 2017). Since the morphological groups that we distinguished showed no marked genetic differentiation (see below) the data on size, age and growth were pooled for both sexes. The few juveniles in the sample remained unsexed and were excluded from the analyses. 


\section{Results}

Out of the candidate nuclear markers two (cnppd and $k d m 3$ ) were dropped because a diagnostic SNP could not be identified and for four (ace, eif4ebp2, ssh2 and syncrip) the Kraken software could not design a suitable set of primers. For the 18 remaining diagnostic nuclear markers for which assays could be designed, PCR amplification for one (amot) failed. The locus slc25 yielded a high frequency of missing data $(16 \%)$ in the reference populations, suggesting it did not amplify the T. ivanbureschi-allele well and results for this marker were also discarded. For the remainder missing data amounted to $1.8 \%$. The four loci ahe, $d d x 17$, dnaj and sre showed highly significant LD for all six pairwise combinations. independence results for the locus ahe (with the fewest missing data) were retained and the others dismissed. Subsequent tests for HW and LD yielded no significancessignificant comparisons under Bonferroni correction. The KASP genotyping output for the remaining 13 markers is summarized in Suppl. Inf. 3. One nuclear SNP marker (col18) showed a single instance of heterozygosity in a parental individual, suggesting either a genotyping error or a marker that is not fully diagnostic. With $\mathrm{N}=82$ (21.7 $\%$ ) of missing data the mtDNA marker (nd4) performed relatively poorly. All newts from the Vlasi population that could be genotyped possessed the mtDNA haplotype typical for T. ivanbureschi, as expected for this marker in this system (for background information see Wielstra et al., 2017a). 
individuals was classified as $\mathrm{F}_{2}$ hybrids $(\mathrm{n}=328,97.6 \%)$. When NewHybrids was instructed to take not two but three generations into account, the majority (99.7\%) was allocated to that third generation. HIest grouped all Vlasi individuals in the middle of the ancestry times heterozygosity bivariate plot, somewhat off-center in the direction of T. ivanbureschi (Figure 34), with the reference populations in the lower left (pure T. macedonicus) and lower right corners (pure T. ivanbureschi). To explore possible differences between morphological groups in the level of estimated ancestry (S) and heterozygosity (H) ANOVA was used to test for differences in $\mathrm{H}$ and S over five (groups 1-5) orand three morphological groups (groups 1, 2-4 pooled, 5) with no statistically significant results $(\mathrm{P}>0.05)$. Pearson correlation coefficients betweenfor S and SVl and the two Hest parameters $\mathrm{H}$ and SVl, were also not significant, for males as well as for females.

The body size distribution showed that females were significantly larger than males (SV1 males $63.9 \mathrm{~mm}, \mathrm{SVl}$ females $67.5 \mathrm{~mm}$; Student's $\mathrm{t}$-test $\mathrm{P}<0.0001$; $\mathrm{SDi}=0.056)$. The age distribution for males and females showed no significant difference (Mann-Whitney $\underline{\mathrm{U}}$-test, $\mathrm{P}>0.05$; Table 2). In both sexes > $70 \%$ of the individuals had an estimated age of 5, 6 or 7 years. Age at maturity estimated by rapprochement was mostly 2,3 or 4 years in both sexes (average males $3.1, n=75$; average for malesfemales 3.0, $\mathrm{n}=74$; Suppl. Inf. 4.). NeThe absence of an observable rapprochement was more frequent in females $(1412.9 \%)$ than in males $(2.6 \%)(P<0.05$, G-test of independence), perhaps suggesting a more regularless drastic shift in resource allocation in the transition from growth to reproduction in females. Longevity was 13 years in males and 11 years in females. The parameters that describe the Von Bertalanffy growth curves and thetheir confidence intervals are presented in Table 3.Suppl. Inf. 5. Results for the Vlasi populations were compared with data from the literature (Cvetković et al., 1996 and A. Ivanović, unpublished data on T. macedonicus and Üzüm, 2006 and Üzüm and Olgun, 2009 on T. ivanbureschi). In three out of four populations females are larger than males of the same age. The other growth curves for populations and species are similar, with no more than $10 \mathrm{~mm}$ difference in SVl among the most different groups (Figure 42B). Unlike T. cristatus x T. marmoratus hybrids, the $T$. macedonicus x T. ivanbureschi hybrids showed no heterosis. Longevity inereased over species aswas
Formatted: Font color: Text 1

Formatted: Font color: Text 1

Formatted: Font color: Text 1

Formatted: Font color: Text 1 Formatted: Font color: Text 1

Formatted: Font color: Text 1

Formatted: Font color: Text 1

Formatted: Font color: Text 1

Formatted: Font color: Text 1

Formatted: Font color: Text 1

Formatted: Font color: Text 1

Formatted: No underline
Formatted: Font color: Auto

Formatted: Font color: Auto 
We encountered a population of crested newts near Vlasi in southeastern Serbia that showed largeextensive phenotypic variation and - being close to the territory of both T. macedonicus and T. ivanbureschi - a hybrid nature was assumed. We were, however, unable to determine the extent of hybridization from morphologymorphological and colouration characters and yet wanted to find out if this hybrid zone population has a unimodal character (with a majority of hybrids and few or no parentals), a bimodal character (with predominantly the parental species and few hybrids), or something in between. We were also interested in fitness consequences that hybridization might have on the demography of the population, in particular if heterosis would be combined with hybrid sterility.

All individuals from the focal population were allocated to the second generation of hybrids, indicating strong admixture along with the absence of recent crossings between the parental species. When a third generation was an option, individuals where allocated to that third generation, further supporting the absence of recent hybrids. Looking yet deeper into the coalescence would require more genetic markers. AncestryAn ancestry versus heterozygosity plotsplot (Figure 4) further support that genotypes in this Vlasi population result from many generations of admixture. In the absence of selection and banningexcluding physical linkage $L D$ halves every next generation and will barely be measurable after a few generations:-(Barton and Gale, 1993). Accordingly, the absence of admixture LD also suggests that hybridization between T. macedonicus and T. ivanbureschi has been going onongoing for more than a couple of generations. hybridized population was also suggested by the absence of genetically diagnosable morphological

239 groups. These results suggest that $T$. macedonicus and $T$. ivanbureschi engage in a unimodal hybrid zone.

240 The genetic affiliation of the Vlasi population is somewhat closer to T. ivanbureschi than to $T$.

241 macedonicus (Figure 34). This is in line with the documented distributions of the two species where the 

2014b). However, the exact position of the center and the width of the cline remain to be documented. This result contrasts with the situation to the northwest, where the two species engage over a wide area of species replacement, including. The mosaic distribution includes a T. ivanbureschi enclave that was cut off from theits main distribution by superseding T. macedonicus (Arntzen, 2003; Arntzen \& Wallis 1999, cf. Figure 1). The sole transmission of the mitochondrial haplotype typical for T. ivanbureschi, that we here confirmed for the Vlasi hybrid population, helped to reconstruct thea scenario in which T. macedonicus advanced at the

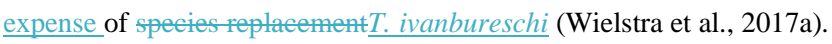

France. Narrow zones with rampantNarrow zones with extensive hybridization are found at contacts between crested newts species belonging to clades B and C (Arntzen et al., 2014; Wielstra et al., 2017b, cf. Figure 1). A relationship appears to exist with species relatedness, so that closely related species form clines and more distant species engage in a mosaic distribution-, like previously described for T. cristatus and T. marmoratus. The presence of a unimodal (clinal) as well as bimodal (mosaic) hybrid zone structure in the T. macedonicus - T. ivanbureschi contact is in line with an intermediate level of relatedness. genetic combinations for testingthat themselves reproduce in nature. Conversely, hybridization in $\underline{a}$ narrow hybrid zone. This result for a comparison of the B and C clades contrasts with the results obtained

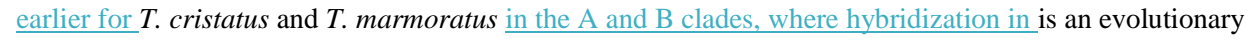
dead-end-because their. These hybrids are largely sterile and introgression is near-absent- (Arntzen \& 
to reproduction. This is more likely to be the case for genetically incompatible than for genetically compatible species. The demonstration of heterosis in any of the six or seventesting grounds for this assertion are several unimodal hybrid zones of closely related Triturus species-, i.e. within the A and B clades (Figure 1) where demonstration of heterosis would contradict this explanationour hypothesis.

\section{Acknowledgements}

We thank R. Bancila and F. Stanescu for help with curve fitting statistics and ${ }_{2}$ O. Schaap and K. Vrieling for running the SNP-line and three anonymous reviewers for helpful comments. BW collected the genetic data as a Newton International Fellow. BW received funding from the European Union's Horizon 2020 research and innovation programme under the Marie Skłodowska-Curie grant agreement No. 655487. MDA and AI were supported by Serbian Ministry of Education, Science and Technological Development grant no. 173043. AI was in receipt of a 'Temminck grant' provided by Naturalis Biodiversity Center.

Permission to collect was obtained from the Ministry of Energy, Development and Envirenmental

Protection of the Republic of Serbia (permit no. $353-01$ 35/2013 08). $\Delta$

\section{References}

Anderson, E. C., \& Thompson, E. A. (2002). A model-based method for identifying species hybrids using multilocus genetic data. Genetics, 160, 1217-1229.

Arnold, M. L. (1992). Natural hybridization as an evolutionary process. Annual Review of Ecology and Systematics, 23, 237-261.

Arntzen, J. W. (2000). A growth curve for the newt Triturus cristatus. Journal of Herpetology, 34, 227 232.

Arntzen, J. W. (2003). Triturus cristatus Superspecies - Kammolch-Artenkreis (Triturus cristatus (Laurenti, 1768) - Nördlicher Kammolch, Triturus carnifex (Laurenti, 1768) - Italienischer Kammolch, Triturus dobrogicus (Kiritzescu, 1903) - Donau-Kammolch, Triturus karelinii 
(Strauch, 1870) - Südlicher Kammolch). Pp. 421-514 in K. Grossenbacher and B. Thiesmeier, eds. Handbuch der Reptilien und Amphibien Europas. Schwanzlurche IIA. Aula-Verlag, Wiebelsheim, Germany.

Arntzen, J. W., Jehle, R., Bardakci, F., Burke, T., \& Wallis, G. P. (2009). Asymmetric viability of reciprocal-cross hybrids between crested and marbled newts (Triturus cristatus and T. marmoratus). Evolution, 63, 1191-1202.

Arntzen, J. W., Smithson, A., \& Oldham, R. S. (1999) Marking and tissue sampling effects on body condition and survival in the newt Triturus cristatus. Journal of Herpetology 33: 567-576.

Arntzen, J. W., \& Wallis, G. P. (1991). Restricted gene flow in a moving hybrid zone of the newts Triturus cristatus and T. marmoratus in western France. Evolution, 45, 805-826.
Formatted: English (United Kingdom)

Formatted: English (United Kingdom)
Arntzen, J. W., \& Wallis, G. P. (1999). Geographic variation and taxonomy of crested newts (Triturus cristatus superspecies): morphological and mitochondrial data. Contributions to Zoology, 68, 181203.

Arntzen, J. W., Wielstra, B., \& Wallis, G. P. (2014). The modality of nine Triturus newt hybrid zones, assessed with nuclear, mitochondrial and morphological data. Biological Journal of the Linnean Society, 113, 604-622.

Barton, N. H., \& Gale K. S. (1993) Genetic analysis of hybrid zones. Pp. 13-45 in: 'Hybrid zones and the evolutionary process' Harrison, R. G. (ed.). Oxford University Press, Oxford, United Kingdom.

Barton, N. H., \& Hewitt, G. M. (1985). Analysis of hybrid zones. Annual Review of Ecology and Systematics, 16, 113-148.

Beysard, M., \& Heckel, G. (2014). Structure and dynamics of hybrid zones at different stages of speciation in the common vole (Microtus arvalis). Molecular Ecology, 23, 673-687.

Cvetković, D., Kalezić, M. L., Djorović, A., \& Džukić, G. (1996). The crested newt (Triturus carnifex) in the submediterranean: reproductive biology, body size, and age. Italian Journal of Zoology, 63, 107-111. 
Dufresnes, C., Bonato, L., Novarini, N., Betto-Colliard, C., Perrin, M., \& Stöck, M. (2014). Inferring the degree of incipient speciation in secondary contact zones of closely related lineages of Palearctic green toads Bufo viridis subgroup. Heredity, 113, 9-20.

Fitzpatrick, B. M. (2012). Estimating ancestry and heterozygosity of hybrids using molecular markers. BMC Evolutionary Biology, 12, 131.

Francillon-Vieillot, H., Arntzen, J. W., \& Géraudie, J. (1990). Age, growth and longevity of sympatric Triturus cristatus, T. marmoratus and their hybrids (Amphibia, Urodela): a skeletochronological comparison. Journal of Herpetology, 24, 13-22.

FSA (2017). Simple Fisheries Stock Assessment Methods. A package for R, available at https://github.com/droglenc/FSA.

Harrison, R. G. (1993). Hybrid Zones and the Evolutionary Process. Oxford University Press, New York, USA.

Jiggins, C. D., \& Mallet, J. (2000). Bimodal hybrid zones and speciation. Trends in Ecology and Evolution, 15, 250-255.

Kearns, A. M., Restani, M., Szabo, I., Schrøder-Nielsen, A., Kim, J. A., Richardson, H. M., ... Omland, K. E. (2018). Genomic evidence of speciation reversal in ravens. Nature Communications, 9, 906.

Lovich, J. E., \& Gibbons, J. W. (1992) A review of techniques for quantifying sexual size dimorphism.

\section{Growth Development and Aging, 56, 269-281.}

Lukanov, S., \& Tzankov, N. (2016). Life history, age and normal development of the Balkan-Anatolian crested newt (Triturus ivanbureschi Arntzen and Wielstra, 2013) from Sofia district. North-Western Journal of Zoology, 12, 22-32.

Maddison, D. R., \& Maddison, W. P. (2005). MacClade: Analysis of Phylogeny and Character Evolution. Sinauer Associates, Sunderland, MA, USA.

Montanari, S. R., Hobbs, J-P. A., Pratchett. M. S., Bay. L. K., \& van Herwerden, L. (2014). Does genetic distance between parental species influence outcomes of hybridization among coral reef butterflyfishes? Molecular Ecology, 23, 2757-2770. 
Nlstools (2015). Tools for Nonlinear Regression Analysis. A package for R, available at https://CRAN.Rproject.org/package=nlstools.

Raymond, M., \& Rousset, F. (1995). GenePop (version 1.2): population genetics software for exact tests and ecumenicism. Journal of Heredity, 86, 248-249.

\section{$\underline{\text { R Core Team (2013). R: A language and environment for statistical computing. R Foundation for }}$}

\section{Statistical Computing, Vienna, Austria.}

SAS Institute Inc. (2011). Base SAS ${ }^{\circledR} 9.3$ Procedures Guide. SAS Institute Inc., Cary, NC, USA.

Schoorl, J., \& Zuiderwijk, A. (1981). Ecological isolation in Triturus cristatus and Triturus marmoratus (Amphibia: Salamandridae). Amphibia-Reptilia, 1, 235-252.

Seehausen, O., Butlin, R. K., Keller, I., Wagner, C. E., Boughman, J. W., Hohenlohe, P. A., ... Widmer, A. (2014). Genomics and the origin of species. Nature Review Genetics, 15, 176-192.

Semagn, K., Babu, R., Hearne, S., \& Olsen, M. (2014). Single nucleotide polymorphism genotyping using Kompetitive Allele Specific PCR (KASP): overview of the technology and its application in crop improvement. Molecular Breeding, 33, 1-14.

Singhal, S., \& Moritz, C. (2013). Reproductive isolation between phylogeographic lineages scales with divergence. Proceedings Royal Society B, 280, 20132246.

Taylor, S. A., Curry, R. L., White, T. A., Ferretti, V., \& Lovette, I. (2014). Spatiotemporally consistent genomic signatures of reproductive isolation in a moving hybrid zone. Evolution, 68, 3066-3081.

Üzüm, N. (2006). Türkiye'deki Triturus karelinii (Strauch 1870) (Urodela: Salamandridae)

Populasyonlarında Yaş Tayini: Populasyonların büyüme, Yaş ve Boy Bakımından Karşılaştırılması. PhD Thesis, Adnan Menderes University, Aydın, Turkey.

Üzüm, N., \& Olgun, K. (2009). Age, size and growth in two populations of the southern crested newt, Triturus karelinii (Strauch 1870) from different altitudes. Herpetologica, 65, 373-383.

Visser, M., de Leeuw, M., Zuiderwijk, A., \& Arntzen, J. W. (2017). Stabilization of a salamander moving hybrid zone. Ecology and Evolution, 7, 689-696. 
Vörös, J., Mikulíček, P., Major, Á., Recuero, E., \& Arntzen, J. W. (2016). Phylogeographic analysis reveals northerly refugia for the riverine amphibian Triturus dobrogicus (Caudata: Salamandridae). Biological Journal of the Linnean Society, 119, 974-991.

\section{Wallis, G. P., \& Arntzen, J.W. (1989) Mitochondrial-DNA variation in the crested newt superspecies:} $\underline{\text { limited cytoplasmic gene flow among species. Evolution, 43, 88-104. }}$

Wielstra, B., \& Arntzen, J. W. (2011). Unraveling the rapid radiation of crested newts (Triturus cristatus superspecies) using complete mitogenomic sequences. BMC Evolutionary Biology, 11, 162.

Wielstra, B., Burke, T., Butlin, R. K., \& Arntzen, J. W. (2017a). A signature of dynamic biogeography: enclaves indicate past species replacement. Proceedings of the Royal Society of London B: Biological Sciences, 284, 20172014.

Wielstra, B., Burke, T., Butlin, R. K., Avcı, A., Üzüm, N., Bozkurt. E., Olgun, K., \& Arntzen, J. W. (2017b). A genomic footprint of hybrid zone movement in crested newts. Evolution Letters, 1, 93 101.

Wielstra, B., Crnobrnja-Isailović, J., Litvinchuk, S. N., Reijnen, B. T., Skidmore, A. K., Sotiropoulis, K. ... Arntzen, J. W. (20132013a). Tracing glacial refugia of Triturus newts based on mitochondrial DNA phylogeography and species distribution modeling. Frontiers in Zoology, 10, 13.

Wielstra, B., Duijm, E., Lagler, P., Lammers, Y., Meilink, W. R. M., Ziermann, J. M., \& Arntzen, J. W. (2014a). Parallel tagged amplicon sequencing of transcriptome-based genetic markers for Triturus newts with the Ion Torrent next-generation sequencing platform. Molecular Ecology Resources, 14, $1080-1089$.

Wielstra, B., Litvinchuk, S. N., Naumov, B., Tzankov, N., \& Arntzen, J. W. (2013b) A revised taxonomy of crested newts in the Triturus karelinii group (Amphibia: Caudata: Salamandridae), with the description of a new species. Zootaxa, 3682, 441-453.

Wielstra, B., Sillero, N., Vörös, J., \& Arntzen, J. W. (2014b). The distribution of the crested and marbled newt species (Amphibia: Salamandridae: Triturus) - an addition to the New Atlas of Amphibians and Reptiles of Europe. Amphibia-Reptilia, 35, 376-381. 
Zuiderwijk, A. (1980). Amphibian distribution patterns in western Europe. Contributions to Zoology, 50, $52-72$.

Suppl. Inf. 1 - Input consensus sequences for Kraken.

Suppl. Inf. 2 - Raw output of the KASP genotyping protocol.

Suppl. Inf. 3 - Genetic data in GenePop format.

Suppl. Inf. 4 - Skeletochronology results along with morphometric data and individual Structure scores. Suppl. Inf. 5 - Parameter values and 95\% confidence intervals (CI) in the Von Bertalanffy growth curves for Triturus populations in the west and southeast of the genus' range.

Figure 1. Distribution of nine Triturus species over Europe and the Near East (after Wielstra et al., 2011). Major clades are A, the marbled newts, B, the $T$. cristatus species group of crested newts and C, the $T$. karelinii species group of crested newts. Note that the spatial contact of clades is limited to central France (clades A and B) and the southern Balkans (B and C). Heterosis in size and longevity was observed in A $\mathrm{x}$ B hybrids (Mayenne, France, white dot, Francillon-Vieillot et al., 1990) and not in B x C hybrids (Vlasi, Serbia, asterisk, present paper). The insert shows the species phylogeny.

Figure 2. Von Bertalanffy growth curves for males and females of the Triturus newts. A - Triturus cristatus (red).,orange), T. marmoratus (green)-2 and Triturus cristatus - T. marmoratus $\mathrm{F}_{1}$ hybrids (black) from Mayenne, France. Data are from Francillon-Vieillot et al. (1990). Females are larger than males of the same age. The hybrids arebecome larger and in general-older than the parental species. No model could be fitted for T. marmoratus females. B - Triturus macedonicus x T. ivanbureschi hybrid population from Vlasi in southeastern Serbia (black lines), T. macedonicus from Montenegro (pink) and T. $\underline{\text { ivanbureschi populations (blue) at localities Keșan and Klaros. Reference data are from Cvetković et al. }}$
Formatted: Font: Bold, Font color: Black

Formatted: Space After: $10 \mathrm{pt}$

Formatted: Font: Not Italic, Pattern: Clear Formatted: Pattern: Clear

Formatted: Subscript 
420

421

425
(1996); Üzüm (2006); Üzüm and Olgun (2009) and A. Ivanović, unpublished data). Females are larger than males of the same age in three out of four populations. The Vlasi populations of hybrids shows no evidence for hybrid vigour in size, growth or longevity. The parameters that describe the Von Bertalanffy growth curves and the confidence intervals are presented in Suppl. Inf. 5.

Figure 3.

\section{Figure 3. Cross-sections of a phalange of a juvenile, male and female Triturus macedonicus x $T$.}

ivanbureschi crested newt from Vlasi. Abbreviataions are: m.c. - marrow cavity, m.1. - metamorphosis

line, e.r. - endosteal resorption, p. - periphery, rap. - rapprochement. Note that the periphery is not

regarded as a LAG. Sections are $18 \mu \mathrm{m}$ thick and taken at the level of the diaphysis. A) Three-year-old

juvenile, SVl $48 \mathrm{~mm}$. Three LAGs (arrow heads) are observed in the periosteal bone as well as a

metamorphosis line (arrow). B) Five-year old male, SVl $64 \mathrm{~mm}$. Four LAGs are observed in the

periosteal bone (arrow heads). Endosteal resorption present (arrow). C) Six-year old female, SVL $64 \mathrm{~mm}$.

Five LAGs are observed in the periosteal bone (arrow heads). Endosteal resorption and rapprochement

are also marked by arrows.

Figure 4. Ancestry versus heterozygosity plot based on 13 species diagnostic nuclear genetic markers.

Individuals from the Vlasi population are shown by open round symbols $(\mathrm{N}=336)$. The left corner of the triangle corresponds to Triturus macedonicus (solid square symbols, $\mathrm{N}=21$ ), the right corner to $T$.

ivanbureschi (solid triangle symbol, $\mathrm{N}=21$ ) and the upper corner to (non-observed) $\mathrm{F}_{1}$ hybrids.
Formatted: Pattern: Clear

Formatted: Subscript 